Nikolai Mikhailov

Università di Pisa

\title{
Eine slowenisch-prußische lexikalisch-mythologische Parallele: kres/kresze
}

\begin{abstract}
Baltoslovanska (prusko-slovenska) leksična in mitološka vzporednica kresze/krệs dokazuje obstoj skupne baltoslovanske osnove *kres- in njeno morebitno uporabo v prabaltoslovanskem mitološkem izrazju.

The Balto-Slavic (Prussian-Slovene) lexical-mythological parallel kresze/krệs proves the existence of a common Balto-Slavic stem *kres- with certain meanings and the possible existence of a primary Balto-Slavic pagan ritual marked by this stem.
\end{abstract}

Die wissenschaftliche Zweckmäßigkeit der Suche nach slowenisch-baltischen Parallelen (insbesondere im lexikalischen Bereich) wurde schon vor fast vierzig Jahren von V. N. Toporov indirekt begründet (Toporov 1958, S. 156). Eine solche Untersuchungs- und Vergleichsarbeit, die im Fall von anderen slawischen Sprachen selbstverständlich (wegen der gemeinsamen parallelen Entwicklung, Arealkontakte usw.) offensichtlicher scheinen kann, hat für das Slowenische eine andere Bedeutung. Die isolierte und marginale (im räumlichen Sinne) Lage der slowenischen Sprache, ihre Umkreisung von meistens nichtslawischen (und schon ganz sicher nicht baltischen) Sprachelementen hat ihren in mehreren Hinsichten konservativen Charakter bedingt. Die Existenz der baltisch-slowenischen exklusiven (und sogar nicht unbedingt exklusiven) Isoglossen kann zu einem der entscheidenden Beweise für die Lösung des Problems der ursprünglichen baltisch-slawischen Einheit und der Rolle einzelner Komponenten innerhalb dieser Einheit werden. Dieses Problem werden wir hier aber nicht besprechen, obwohl es noch nicht endgültig gelöst ist, wie die ständig weitergehenden polemischen Diskussionen zeigen (vgl. z. B. Toporov 1988 »contra Trubačev 1983, Trubačev 1993 »contra« Toporov 1988 u. a.).

Konkret hat sich mit dem Problem der baltisch-slowenischen Parallelen F. Bezlaj beschäftigt (vgl. Bezlaj 1967; Bezlaj 1968; Bezlaj 1974; Bezlaj 1977; Bezlaj 1981). Das baltische Material wurde natürlich auch in den drei bis jetzt veröffentlichten Bänden des Etimološki slovar slovenskega jezika von F. Bezlaj (Bd. I-II) und von seinen Nachfolgern M. Snoj und M. Furlan (Bd. III) benutzt. Einige Bemerkungen zu diesem Thema sind von W. Boryś gemacht worden (Boryś 1992a, Boryś 1992b). Ein Artikel, in dem mehrere baltisch-slowenische sprachlich-mythologische Analogien angeführt werden, ist vor kurzem im italienischen Jahrbuch Res Balticae erschienen (Mikhailov 1996b).

In letzter Zeit beschränkt sich das »baltisch-slawische Problem« nicht nur auf die Aspekte der Sprachentwicklung. Es handelt sich jetzt um eine komplexere Vision des ganzen baltisch-slawischen Materials, welche auch die Analyse der Ethnogenesis, 
der vermutlich gemeinsamen Mythologie und einiger Riten einschließt. Als ein Beispiel einer solchen Analyse (von den sprachlichen Daten zu den mythologischen Interpretationen) mit der Benützung des slowenischen Materials könnte man die von F. Bezlaj vorgeschlagene, äußerst interessante sprachlich-mythologische Parallele slowen. Kúrent/pruß. Curche anführen (Bezlaj 1981; Bezlaj 1982, S. 112-113; vgl. darüber auch Matičetov 1985 = Matičetov 1989; Mikhailov 1994; Mikhailov 1996b).

Eine andere Parallele dieser Art, die auch zu bestimmten mytho-rituellen Rekonstruktionen führen könnte, ist pruß. kresze und slowen. krệs (- kresnice - Krệsnik). Auf der sprachlichen Ebene ist diese Parallele nicht exklusiv (vgl. slaw. *krěsb $1 * k r b s b$ und die Realisierung dieses Stammes in anderen slawischen Sprachen), wenn man sie aber in einer gewissen rituellen Perspektive analysiert, könnte sie zu einigen interessanten Schlüssen führen.

Der Bischof Michael Junge von Samland (1425-1442) hat einige Anmerkungen über die Reste des alten heidnischen Glaubens bei den alten Prußen hinterlassen. In dem für seine Diözese geschriebenen Mandat Articuli per Prutenos tenendi et erronei contra fidem abiciendi erwähnt er auch Kresze, anscheinend ein heidnisches Fest: »Item ut de cetero in siluis aut nemoribus nullas faciant congregaciones seu celebritates contra statuta sancte matris ecclesie, et eorum $\mathrm{K} \mathrm{r}$ e s z e amplius non celebrent sub pena rigide correccionis et priuacionis ecclesiastice sepulture « (zit. nach Mannhardt 1936, S. 158; vgl. Toporov 1984, S. 177 ff.).

Dasselbe Fest betreffen anscheinend zwei andere Zeugnisse aus dem XV. Jh. Sein Name erscheint aber verändert in zwei verschiedenen Formen. In den Frauenburger Beschlüssen (= Landesordnung für das ganze Ordensland) wurde am 22. Mai 1445 geschrieben: »Wer sie dorobir wird leyden, sal nicht vor eynen guten cristen gerichtet werden, und sunderlich die Preusen, das die ablegen heydensche weise als an clederen, heiligunge des fyhes und des byers, und alle unortliche getrencke, das uff Zameland die k e y s e und dy mettele is genant " (Mannhardt 1936, S. 168). In diesem Fall geht es wahrscheinlich um einen Schreibfehler: das $r$ ist weggelassen worden (keyse statt *kreyse).

Bekannt ist auch eine Klageschrift des Propstes von Samland über das Verderben von Samländern: „Obir das haben sie etliche heidenische qwasse, als metle, k r y s z e, snyke und dergleich, domete sie viele czeit vorthun und vorczeren domete was sie haben« (Mannhardt 1936, S. 168).

Man kann vermuten, daß eine von den drei registrierten Formen (kresze, keyse, krysze) und zwar keyse falsch geschrieben worden ist. Deswegen könnte man annehmen, daß das $r$ in diesem Wort anwesend sein muß und daß die falsch geschriebene Form keyse als *kreyse rekonstruiert werden kann. Nesselmann schlägt vor, diese Form auf *kerse zurückzuführen (Nesselmann 1873, S. 80), was weniger begründet scheint, sogar wenn man eine Metathesis *kerse $<* k r e s e$ vermutet.

Hinsichtlich der Etymologie dieses prußischen Wortes herrscht noch keine Klarheit. Es gibt drei wichtigste Hypothesen. Die erste spiegelt die Meinung von Milewski wider, daß das ursprünglich kein echtes prußisches Wort sei, sondern eine sehr frühe Entlehnung (IX. Jh.) aus dem nicht registrierten (»niezachowani«) altpolnischen *krzas (Milewski 1947; darüber s. auch Toporov 1984, S. 178). Tatsächlich müßte das echte baltische (prußische) Wort eine andere Form haben, vgl. das rekon- 
struierte balt.-sl. *kraip-s-/*kreip-s-/*krīp-s- und lit. kreĩpti 'wenden, biegen', kraipý$t i, k r y \tilde{p} t i$ 'sich biegen, abbiegen' (Toporov 1984, S. 178; vgl. auch Bezlaj 1982, S. 90 im Verhältnis zu dem slow. krệs als eine mögliche, aber nicht sichere Parallele). Die Interpretation von Milewski hat einen Grund, weil die echte baltische Form anders sein müßte. Andererseits scheint uns die Theorie der Entlehnung eines nicht registrierten altpolnischen Wortes auch in chronologischer Hinsicht nicht besonders überzeugend. Man könnte auch daran erinnern, daß einige Hypothesen von Milewski schon manchmal kritisiert worden sind (vgl. z. B.: Smoczyński 1992).

Die z w e i t e Hypothese »arbeitet« mit der »korrigierten« Form *kreyse bzw. *kreysze, die dem baltischen Vokalismus näher steht. Das Ausfallen von dem im Baltischen angeblich anwesenden - $p$ - bleibt aber nicht geklärt (Toporov 1984, S. 178-179). Man könnte eine spätere Eliminierung von - $p$ - aus der Kombination der Konsonanten -psz- vermuten: *kreyse $<*$ kreysze $<*$ kreyp-s(z)e, angenommen, daß $s z$ zwei Laute bezeichnete. Das ist aber eher unwahrscheinlich, weil $s z$ in der deutschen Übertragung vermutlich doch wie [s] ausgesprochen wurde und weil uns zur Zeit keine anderen Fälle der Eliminierung von - $p$ - vor einer Gruppe von Konsonanten bekannt sind. Äußerst interessant und verführerisch ist die Rekonstruktion Vasmers für das Slawische $\left(* k r e ̌ p s o-,{ }^{*} k r b p s o-\right)$, die er mit den baltischen Beispielen verbindet, aber leider nicht ausführlich kommentiert (Vasmer 1986, S. 372-373).

Die dritte Hypothese beruht auf der Beziehung zwischen kresze und slaw. *krěs-, die aber zu dem im lit. krósnis und lett. krâsns 'Ofen' anwesenden Stamm zurückführt (Toporov 1984, S. 179 schließt das nicht aus). Ein solcher Vergleich ist von Vasmer 1986, S. 373 und dann von Bezlaj 1982, S. 90 abgelehnt worden.

Neben diesen drei Hypothesen wäre es interessant, auch die Meinung von Mažiulis zu erwähnen. In seinem Prūsu kalbos etimologijos žodynas führt Mažiulis das Lexem kresze nicht an. Unter creslan 'Lehnstuhl' spricht er aber über den baltisch-slawischen Stamm *kres-/*krēs-/*kras- 'sukti, lenkti' = 'drehen, biegen' (Mažiulis 1993, S. 268-270; anders über creslan Toporov 1984, S. 179-183). Die Existenz dieses Stammes im Baltisch-Slawischen und eine solche Bedeutung von ihm, die, wie man gleich sehen wird, einer der Bedeutungen des slaw. *krěs- entspricht, könnte auch die ursprüngliche baltisch-slawische Natur des prussischen kresze beweisen. Man kann vermuten, daß, wenn Mažiulis kresze in sein Wörterbuch eingeschlossen hätte, er dieses Lexem zu dem seiner Meinung nach vorhandenen balt.-slaw. Stamm *kres-/*krēs-/*kras- zurückgeführt hätte.

Auf jeden Fall wird in keiner dieser drei Hypothesen die Beziehung zwischen pruß. kresze und slaw. *krěs- ausgeschlossen. Mažiulis führt kein späteres slawisches Material an, spricht aber über den baltisch-slawischen Stamm, was automatisch auch die weitere Entwicklung des Stammes im Slawischen voraussieht. Die Etymologie des slawischen Stammes ist auch nicht volkmommen klar (vgl. Bezlaj 1982, S. 90: »etimologija ni jasna «). Auf der semantischen Ebene ist dieser Stamm im Slawischen (aber auch im Indogermanischen) mit den Begriffen von 1) 'Feuer', 2) 'Wende' ('Sonnenwende')/'Auferstehen'/'Wiedergeburt' und 3) 'Schlagen' verbunden (Toporov 1984, S. 178-179; teilweise auch ESSJa 1985, SS. 124-125; Vasmer 1986, SS. 372-373). Für den ersten sprechen so bekannte Beispiele wie rus. kresit', kresát', sbkr. krèsati, slowen. kresáti 'excudere ignem', krệs 'ogenj pred 24. junijem' u. a.; für den zweiten aksl. vbskrěsiti 'wiedererwecken, neu beleben' mit weiteren slawischen Formen; für 
den dritten wieder slowen. kresáti mit der zweiten Bedeutung 'sekati, obsekavati, tesati', mit der perfektiven Form krésniti 'udariti' (Bezlaj 1982, 90). Skok spricht sogar über den Zusammenhang kres - kremen (Skok 1972, S. 190-191, vgl. die Bemerkung von Trubačev über die Erklärung von Otrębski in Vasmer 1986, S. 373). Noch früher sprach Pokorny über den indogermanischen Stamm *krek- 'schlagen' und führte mehrere Beispiele aus slawischen Sprachen mit der Bedeutung '(mit dem Feuerstahl) Feuer schlagen' (Pokorny 1959, S. 618-619) an. Andererseits sind diese

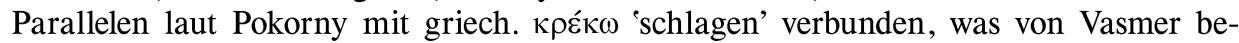
zweifelt worden ist (Vasmer 1986, S. 373).

Die slowenischen Daten in bezug auf pruß. kresze sind besonders bedeutsam, in gewissem Sinne sind sie wichtiger und interessanter als die Daten anderer slawischer Sprachen. Man kann das aus folgenden Gründen behaupten:

1) auf der semantisch-lexikalischen Ebene ist der slowenische Stamm kres- sowohl mit dem Begriff von Feuer, vgl. kresáti 'excudere ignem', als auch mit den Begriffen 'wegschneiden, abhacken' und 'schlagen' (kresáti, krésniti, krẹsiti se iskriti, lesketati') und mit dem Begriff des »neuen Lebens« (vgl. krẹsen 'živahen, močan' usw.) verbunden (vgl. auch krêsâti »elicere ignem, laedere pedem« in Pohlin, Bezlaj 1982, S. 90);

2) auf der mythologisch-rituellen Ebene ist für die Slowenen das ursprünglich heidnische Fest und Ritual krệs (genau mit diesem Namen! - und nur sekundär Šentjanževo, Ivanje genannt -) besonders wichtig (vgl. Kuret 1989, S. 383 ff. und mehrere andere Forschungen). In anderen slawischen Traditionen wird dieses Fest schon lange mit anderen und zwar späteren christlichen Namen bezeichnet.

3) Nur in der slowenischen Tradition gibt es ein mythologisches Wesen, in dessen Namen man auch den Stamm kres- findet, und zwar Krệsnik (vgl. aber SP 1962, S. 382: Kresník). Wenn man die Hypothese akzeptiert, daß Krệsnik eine slowenische Hypostase des gemeinsamen baltisch-slawischen Donnergottes ist (Mikhailov 1996), wird ganz offensichtlich, daß gerade die slowenische Sprache die sakrale archetypische Bedeutung dieses Stammes behalten hat (vgl. den Kresnik, der von oben nach unten mit dem Feuer / mit den Blitzen seinen chthonischen Gegner schlägt usw.). Gleichzeitig muß man präzisieren, daß die von Krek vorgeschlagene, von Kelemina wiederholte und von Bezlaj abgelehnte Analogie: altruss. Chors - slaw. *krěs-, welche für eine originelle mythologische Interpretation verführerisch wäre, doch nicht akzeptabel ist (Krek 1887, S. 393-394; Kelemina 1930, S. 8; Bezlaj 1951, S. 346; vg1. auch die letzten Daten über Chors: Toporov 1995).

Man kann vermuten, daß das prußische Fest kresze (abgesehen von der Frage, ob das eine sehr frühe Entlehnung aus dem Slawischen oder eine baltische Realisierung eines gemeinsamen Urstammes ist) dem urslowenischen heidnischen Fest kres entspricht. Außer der Form des Wortes sprechen mehrere Parallelen und Beweise dafür. Vergleichbar sind z. B. das Feiern von kresze »in silvis in nemoribus « und die Bedeutung 'sekati, obsekavati, tesati' '(Zweige) wegschneiden' (für das rituelle Feuer?) von slowen. kresáti. Das slowenische Material zeigt, daß krệs, kresovânje ein hochentwickeltes und auf eine bestimmte Art organisiertes heidnisches Ritual war. Die slowen. kresnice bzw. ladarice können als Opferpriestinnen bei der Kres-Feier interpretiert werden und ihr koledovanje als ein rituelles Gebet. 
Der Zusammenhang zwischen pruß. kresze und slowen. krệs - kresníce Krệsnik erlaubt, bestimmte Schlüsse für mehrere Forschungsbereiche zu ziehen.

Für das Prußische könnte man aufgrund der slowenischen Parallele vermuten, daß kresze auch bei den Prußen das heidnische Fest der Sonnenwende war und möglicherweise auch mit der konventionellen Figur der Donnergottheit verbunden ist.

Für das Slowenische beweist diese Parallele die wichtige Rolle und den Archaismus der slowenischen Daten sowohl für die sprachlich-semantische als auch für die mythopoetische Rekonstruktion einiger bedeutsamer sakraler Begriffe.

Für das Baltisch-Slawische erlaubt der Zusammenhang krệs/kresze über das Konzept der »baltisch-slawischen Mythologie« und des gemeinsamen rekonstuierbaren Rituals zu sprechen. Angenommen, daß kresze und krệs dasselbe archaische heidnische Sonnenwendfest bezeichnen, könnte man folgende Vermutungen anführen:

Wenn die zweite Hypothese der Etymologie von kresze stimmt (ein baltisch-slawischer Urstamm mit dem späteren Ausfallen des - $p$ - im Slawischen), ist die oben analysierte Parallele ein gültiger Beweis der Realisierung dieses Stammes in verschiedenen Traditionen, die doch eine gemeinsame Herkunft haben.

Sogar wenn man die übliche, aber unserer Meinung nach ziemlich unsichere erste Hypothese akzeptiert (ein slawisches [altpolnisches] Lehnwort im Prußischen, welches vor dem Ende des IX. Jh. ins Prußische eingedrungen ist), wird diese Analogie sekundär, bleibt aber doch gültig. Das heißt, daß das slowenische Wort (wie man gut weiß, bevölkerten die Urslowenen schon lange vor dem IX. Jh. die mitteleuropäischen Gebiete) aus dem Urslawischen stammt und am besten auch die urslawische sakrale Bedeutung widerspiegelt, während das prußische Wort denselben urslawischen Stamm erst später benutzt hat.

Die mythologisch-rituelle Analyse erlaubt doch zu vermuten, daß slowen. krệs und pruß. kresze die Realisierung desselben sakralen Begriffes in verschiedenen Traditionen darstellen, insbesondere wegen mehrerer primärer Bedeutungen, die das Element *kres- in der slowenischen Sprache behalten hat, und wegen des archaischen Charakters des slowenischen Festes im Vergleich zu anderen slawischen Traditionen, die sich im Territorium zwischen Slowenen und Prußen befinden.

Prispelo maja 1996, sprejeto decembra 1996

Received May 1996, accepted December 1996

\section{Literatur}

Bezlaj $1951=$ F. Bezlaj, Nekaj besedi o slovenski mitologiji $\mathrm{v}$ zadnjih desetih letih, Slovenski etnograf, III-IV, 1951, S. 342-353.

Bezlaj 1967 = F. Bezlaj, Eseji o slovenskem jeziku, Ljubljana 1967.

Bezlaj $1968=$ F. Bezlaj, Einige slovenische und baltische lexikalische Parallelen, Linguistica, VIII, 1966-68, S. 63-81.

Bezlaj $1974=$ F. Bezlaj, Spuren der baltoslawischen Wortmischungen, Baltistica, X(1), 1974, S. 21-30.

Bezlaj 1977 = F. Bezlaj, Slovensko-baltijskie leksičeskie paralleli, Baltistica, II priedas, 1977, S. 15-19. 
Bezlaj 1981 = F. Bezlaj, Sloveno-baltica, Jezik in slovstvo, 2, 1980-81, S. 51-53.

Bezlaj 1982 = F. Bezlaj, Etimološki slovar slovenskega jezika, II, Ljubljana 1982.

Boryś 1992a $=\mathrm{W}$. Boryś, Z nawiązań leksykalnych południosłowiańsko-bałtyckich: sch. i słń. ježa = lit. ežià, łot. eža, in W. Smoczyński, A. Holvoet (Hrsg.), Colloquium Pruthenicum Primum, Warszawa 1992, S. 129-134.

Boryś $1992 \mathrm{~b}=\mathrm{W}$. Boryś, $\mathrm{Zu}$ den südslawisch-baltischen lexikalischen Verknüpfungen, Linguistica Baltica, 1, 1992, S. 193-200.

ESSJa 1985 = Ėtimologičeskij slovar' slavjanskich jazykov. Praslavjanskij leksičeskij fond. Vypusk 12. Pod redakcijej O. N. Trubačeva, Moskva 1985.

Kelemina 1930 = J. Kelemina, Bajke in pripovedke slovenskega ljudstva, Celje 1930.

Krek $1887=$ G. Krek, Einleitung in die slavische Literaturgeschichte, Graz 1887.

Kuret 1989 = N. Kuret, Praznično leto Slovencev, I, druga izdaja, Ljubljana 1989.

Mannhardt 1936 = W. Mannhardt, Letto-preußische Götterlehre, Riga 1936.

Matičetov $1985=$ M. Matičetov, O bajnih bitjih Slovencev s pristavkom o Kurentu, Traditiones, 14, 1985, S. 88-100.

Matičetov 1989 = M. Matičetov, O mifičeskich suščestvach u slovencev i special'no o Kurente, in: Slavjanskij i balkanskij fol'klor, Moskva 1989, S. 88-100.

Mažiulis 1993 = V. Mažiulis, Prūsų kalbos etimologijos žodynas, 2, I-K, Vilnius 1993.

Mikhailov $1994=$ N. Mikhailov, Ešče raz o slovenskom Kurente. Nekotorye paralleli, in: Balkanskie čtenija - 3, Moskva 1994, S. 119-123.

Mikhailov 1996a $=$ N. Mikhailov, Fragment slovenskoj mifopoètičeskoj tradicii, in: Koncept dviženija. Struktura slovesnych i neslovesnych tekstov slavjanskoj tradicii, Moskva 1996, S. 127-131.

Mikhailov 1996b $=$ N. Mikhailov, Baltico-slovenica. Alcuni paralleli mitologici, Res Balticae, 2, 1996, S. 151-178.

Milewski $1947=$ T. Milewski, Stosunki językowe polsko-pruskie, Slavia Occidentalis, XVIII, 1947, S. 21-84.

Nesselmann $1873=$ G. H. F. Nesselmann, Thesaurus linguae Prussicae. Die preussische Vokabelvorrath soweit derselbe bis jetzt ermittelt worden ist, Berlin 1873.

Pokorny 1959 = J. Pokorny, Indogermanisches etymologisches Wörterbuch, I, 2. Aufl., Bern-Stuttgart 1959.

Skok 1972 = P. Skok, Etimologijski rječnik hrvatskoga ili srpskoga jezika, 2, Zagreb 1972.

Smoczyński $=1992 \mathrm{~W}$. Smoczyński, O tzw. zapożyczeniach prapolskich $\mathrm{w}$ języku staropruskim, in: Słowiańskie pogranicza językowe, Warszawa 1992.

SP 1962 = Slovenski pravopis, Ljubljana 1962.

Toporov 1958 = V. N. Toporov, Slovenica, Slavistična revija, XI, 3-4, 1958, S. $156-168$

Toporov 1984 = V. N. Toporov, Prusskij jazyk. Slovar'. K-L, Moskva 1984.

Toporov 1988 = V. N. Toporov, K rekonstrukcii drevnejšego sostojanija praslavjanskogo, in: Slavjanskoe jazykoznanie. X meždunarodnyj s"ezd slavistov, Sofija, sentjabr’ 1988 g. Doklady sovetskoj delegacii, Moskva 1988, SS. 264-292. 
Toporov $1995=$ V. N. Toporov, Ob iranskom mifologičeskom èlemente v drevnerusskoj kul'ture. I. Ešče raz o Chorse i Simargle, in V. N. Toporov, Svjatost' i svjatye $\mathrm{v}$ russkoj duchovnoj kul'ture, I. Pervyj vek christianstva na Rusi, Moskva 1995, SS. 512-526, 567-574.

Trubačev $1983=$ O. N. Trubačev, Jazykoznanie i ètnogenez slavjan. Drevnie slavjane po dannym ètimologii i onomastiki, in Slavjanskoe jazykoznanie. IX meždunarodnyj s"ezd slavistov. Doklady sovetskoj delegacii, Moskva 1983, SS. 231-270.

Trubačev 1993 = O. N. Trubačev, Etnogenez i kul'tura drevnejšich slavjan, Palaeoslavica, 1, 1993, SS. 9-40.

Vasmer 1986 = M. Vasmer, Etimologičeskij slovar' russkogo jazyka, perevod s nemeckogo i dopolnenija O. N. Trubačeva, 2, II-oe izd., Moskva 1986.

\section{Slovensko-pruska leksikalno-mitološka vzporednica: $k r e s / k r e s z e$}

$\mathrm{V}$ treh nemških virih iz 15. stol., ki se nanašajo na baltske (v glavnem pruske) poganske šege, je omenjen Kresze 'neki poganski praznik' (drugi dve obliki sta keyse, krysze). Obliko te besede in njen pomen lahko primerjamo s slovanskim *krěs b in torej s slovenskim krệs 'ogenj pred 24. junijem', kar prvotno pomeni tudi poganski praznik. Etimologija Kresze ni popolnoma jasna, ker je prvotna baltska beseda morala imeti drugo obliko. Običajno domnevajo, da je prus. Kresze zelo zgodnja slovanska (staropoljska?) izposojenka. Poleg nje obstajata še dve domnevi, ki sta manj verjetni, toda nikdar nista bili dokončno zavrnjeni.

Vzporednica kresze/kres je zanimiva predvsem na mitološko-ritualni ravni. Lahko domnevamo, da prusko kresze ustreza slovenskemu poganskemu ritualu krệs, kresovânje. Taka paralela lahko dokazuje prvotno sakralnost elementa *kres- na vsem baltoslovanskem območju, poleg tega potrjuje tudi arhaičnost slovenščine, v kateri je podstava *kres- ohranila več prvotnih pomenov, kakor $\mathrm{v}$ drugih slovanskih jezikih.

\section{A Slovene-Prussian Lexical-Mythological Parallel: kres/kresze}

In three 15th-century German sources concerning certainly Baltic (chiefly Prussian) pagan customs, the term Kresze 'a pagan feast' is mentioned (the other two forms mentioned are keyse, krysze). This form is comparable to the Slavic *krěs'b and also with the Slovene krệs 'ogenj pred 24. junijem', which means primarily a pagan feast. The etymology is not fully clear, because the ancient Baltic word must have had an other form. The traditional hypothesis considers the Prussian Kresze as an earlier Slavic (Old-Polish?) lexeme. Two other etymological explanations of this word are less certain, but they have never been definitively refuted.

The parallel kresze/krẹs is interesting especially on a mythological and ritual level. We may assume that the Prussian kresze corresponds to the Slovene pagan ritual krệs, kresovânje. Such a parallel would prove the primary sacred character of the stem *kres- for all the Balto-Slavic area and confirm the theory that the Slovene form is archaic, where the stem *kres- has maintained more of its primary meanings than in other Slavic languages. 